\title{
Localization of QTL for cold tolerance at the germination stage of rice (Oryza sativa L.) using recombinant inbred lines
}

\author{
Liangzi Cao - Shukun Jiang - Guohua Ding - Tongtong Wang • \\ Liangming Bai · Jinsong Zhou $\cdot$ Yu Luo - Tianshu Xia · Hui Jiang • \\ Kai Liu $\cdot$ Xueyang Wang $\cdot$ Guang Yang $\cdot$ Shichen Sun
}

Received: 19 March 2021 / Accepted: 21 December 2021 / Published online: 10 January 2022

(C) The Author(s) 2022

\begin{abstract}
The cold tolerance of germinating directsown rice (Oryza sativa L.) has an increased rate of emergence, which ensures vigorous seedling growth. Research on QTL localization for cold tolerance at the germination stage can assist in molecular markerassisted selection and enhance breeding efficiency. In this study, 94 populations of recombinant self-incompatible lines from Heigu and $\mathrm{Ha} 9366$ were selected to investigate germination rates at low temperatures. It was found that two QTL loci (qLTG-3 and qLTG-12) were located at different germination times on chromosomes 3 and 12, respectively. The two QTLs at three different germination times, located using QTL, accounted for 21.3-25.9\% of the phenotypic variation. Moreover, a reciprocal effect was detected between
\end{abstract}

L. Cao

Postdoctoral Science Research Workstation of

Heilongjiang Academy of Agricultural Sciences,

Harbin 150086, China

L. Cao $\cdot$ S. Jiang $\cdot$ G. Ding $\cdot$ T. Wang $\cdot$

L. Bai · J. Zhou - Y. Luo - T. Xia .

G. Yang $\cdot$ S. Sun $(\square)$

Institute of Tillage and Cultivation, Heilongjiang

Academy of Agricultural Sciences, 250088 Harbin, China

e-mail: liangzi0219@hotmail.com

L. Cao $\cdot$ S. Jiang $\cdot$ G. Ding $\cdot$ T. Wang $\cdot$

L. Bai $\cdot$ J. Zhou $\cdot$ S. Sun

Heilongjiang Rice Quality Improvement and Genetic

Breeding Engineering Research Center, 250086 Harbin,

China the two QTLs. The double QTLs increased the germination rate by $22-27 \%$ in this population. Additionally, qLTG-12 improved cold tolerance at the seedling stage. The results of this study might provide the materials and molecular markers for future molecular marker-assisted breeding for cold tolerance at the germination stage.

Keywords Rice Cold tolerance at germination stage $\cdot$ QTL · Direct seeding

\footnotetext{
H. Jiang · K. Liu

Heilongjiang Academy of Agricultural Sciences,

Harbin 150086, China

X. Wang

Soybean Research Institute, Heilongjiang Academy of Agricultural Sciences, 250086 Harbin, China
} 


\section{Introduction}

Rice (Oryza sativa L.) is one of the major food crops and a staple food for over 50\% of the world population. Asia is responsible for more than $80 \%$ of global rice consumption, and China is the largest (30\%) rice producer and consumer in the world (http:// ricepedia.org). The northeast region is one of the leading rice-producing regions in China. Additionally, it is also classified as a high-latitude cold-region rice cultivation area along with Hokkaido, Japan. "Dry breeding at lower planting density" is the dominant plantation strategy in cold areas that grow rice at high latitudes. Using this strategy, strong seedlings were cultivated that reduced the damage caused by weeds (Farooq et al. 2011; Olajumoke et al. 2016; Singh et al. 2001). The economic development of China has reduced the rural labor force and increased labor costs, which have dramatically increased the cost of growing rice through the "Dry breeding at lower planting density" planting strategy. Contrastingly, the direct seeding method reduces seedling field, water, labor, and effort (Feng et al. 2020; Ya et al. 2020; Zhang et al. 2015; Zhen 2015). This method not only significantly reduces the cost of rice cultivation but also significantly alleviates labor shortages. However, due to frequent occurrences of low-temperature damage in the spring in cold regions at high latitudes, rice that can germinate and grow at low temperatures is required for direct seeding. Unfortunately, the existing rice varieties cannot germinate well at low temperatures, which adversely affects the development of directseeded rice (Cao et al. 2018; Chen et al. 2006).

Many low-temperature germination-related QTLs are located on the 12 chromosomes; however, only a few can be used for molecular-assisted breeding. Currently, only $q L T G-3$ has been cloned (OsO3 g0103300), which is the only gene related to lowtemperature germination (Fujino et al. 2008). In this study, 94 recombinant inbred lines (RILs) from Heigu and Ha 9366 were used to perform the QTL analysis on low temperature-resistant germination traits, which might provide a theoretical basis for further fine positioning and the development of molecular markers for molecular marker-assisted breeding (MAS).

The cold tolerance of rice at the germination stage is mainly identified by its ability to germinate at low temperatures, which is evaluated by identifying the seedling vigor and viability at low temperatures. The low-temperature germination of crops is a quantitative trait controlled by multiple genes. The QTLs associated with low-temperature germination have been detected on all 12 chromosomes. There are 97 QTLs associated with cold tolerance at the germination stage. More QTLs were detected on chromosomes 3, 5, 7, and 11, and fewer on chromosomes 2, 9, and 12 (Sheng et al., 2001; Fujino et al. 2004; Chen and Li 2005; Qiao et al. 2005; Han et al. 2007; Li et al., 2008; Gong et al. 2009; Li et al. 2011; Yang et al. 2016; Jiang et al. 2017; Yang et al. 2018) (Table 1).

Although many QTLs associated with low-temperature germination have been localized on the 12 chromosomes, the number of molecular markers, as well as, the information that can facilitate the screening of suitable direct-seeded rice varieties with cold tolerance, is insufficient. In this study, 94 recombinant inbred lines (RILs) and 32 advanced generation lines of Heigu and Ha 9366 were used for QTL analysis and to evaluate the number of QTLs associated with cold tolerance during germination. We aimed to provide a theoretical basis for further fine localization and development of molecular markers for molecular marker-assisted breeding (MAB).

Table 1 QTLs related to cold tolerance by germination stage in rice

\begin{tabular}{lc}
\hline Chromosome & Germination stage \\
\hline 1 & 5 \\
2 & 4 \\
3 & 11 \\
4 & 6 \\
5 & 18 \\
6 & 7 \\
7 & 15 \\
8 & 5 \\
9 & 4 \\
10 & 8 \\
11 & 11 \\
12 & 3 \\
Total & 97 \\
\hline
\end{tabular}




\section{Materials and methods}

Experimental materials

The LTH/H9366 recombinant inbred line population, consisting of 94 lines, was obtained by the single seed transmission method using the low-temperature germination variety Heigu (LTH) and the conventional high-latitude cold rice variety $\mathrm{Ha} 9366$ (H9366) as parents. The advanced generation lines are rice varieties suitable for cultivation in the Heilongjiang Province, China, above generation F9.

In 2017, two parents and 94 recombinant inbred lines were planted in the paddy field base of China Heilongjiang Academy of Agricultural Sciences Democracy Park (E: 126.8, N: 45.4). The planting density is 4 inches $* 8$ inches, sowing in mid-April, harvesting in late September. After harvest, seeds were germinated at low temperature.

Low-temperature germination test

After the parents and population materials were harvested at maturity, we interrupted dormancy at $50{ }^{\circ} \mathrm{C}$ for seven days. Fifty filled seeds were randomly selected from each strain and placed in a $9 \mathrm{~cm}$ Petri dish lined with two layers of filter paper after disinfection. Each Petri dish was filled with $10 \mathrm{~mL}$ of sterile water and incubated at $15^{\circ} \mathrm{C}$ in an incubator for germination. Three replications were performed for each line and parent. The number of germinated grains was recorded, and the germination rates were calculated on the fifth, sixth, and seventh day after germination, and the germination rate was calculated. Germination rate $(\%)=($ total number of germinations 5,6 , and 7 days later)/50 grains. Successful germination was determined by the radicle or germ length $>1$ $\mathrm{mm}$. After seven days of low-temperature treatment, the seeds were transferred to an incubator at $30^{\circ} \mathrm{C}$, and the germination rate was tested after four days. A seed germination rate above $80 \%$ indicated that the low-temperature germination ability was not affected by secondary dormancy (refer to partial method modifications by Jang et al., 2006).

\section{Marker analysis}

The CTAB method introduced by Murray and Thompson (1980) was used as a reference to extract the DNA of each strain. Parent screening was conducted for 350 SSR markers, and 125 SSR markers were finally selected for marker analysis. The PCR reaction volume was $20 \mu \mathrm{L}$, which included $1 \mu \mathrm{L}$ of 5 $\mu \mathrm{mol} / \mathrm{L}$ primers, $1 \mu \mathrm{L}$ of DNA, and $2 \times$ TaqMix. The PCR conditions were: pre-denaturation at $94{ }^{\circ} \mathrm{C}$ for $5 \mathrm{~min}$, denaturation at $94{ }^{\circ} \mathrm{C}$ for $30 \mathrm{~s}$, renaturation at 52-60 ${ }^{\circ} \mathrm{C}$ for $30 \mathrm{~s}$, and extension at $72{ }^{\circ} \mathrm{C}$ for $1 \mathrm{~min}$, with a total of 35 cycles. Then, a final extension was performed at $72{ }^{\circ} \mathrm{C}$ for $5 \mathrm{~min}$, and the products were stored at $4{ }^{\circ} \mathrm{C}$. The PCR products were electrophoretically separated on a $4 \%$ agarose gel and then detected using an imaging system.

Construction of linkage mapping and QTL analysis

Mapmaker 3.0 and WinQTLCart 2.5 software were used for the QTL analysis of low-temperature germination traits of rice. Mapmarker 3.0 was used to construct a genetic linkage map and obtain the genetic linkage centimorgan (cM) between markers (Fig. 3); Win-QTLCart 2.5 was used to generate LOD curves to determine QTL loci and their distribution on the chromosomes. During the analysis, the effects of the main-effect markers and the interaction markers were controlled simultaneously. The likelihood ratio test and the t-test were used in combination to test the significance of the effect of QTL $(P \leq 0.005)$ and interaction effect $(P \leq 0.001)$, as well as for calculating the contribution rate.

Analysis of the utilization value of the QTL locus

Thirty-five rice lines were genotyped using SSR primers near the QTL. The method was similar to that described in "Marker analysis" section. The cold tolerance at the germination stage was determined as described in "Low temperature germination test" section. The cold tolerance at the germination stage was investigated using plants that had grown to the two-leaf stage by treating them at $5{ }^{\circ} \mathrm{C}$ for five days, and after treatment, the phenotype was investigated after five days of recovery under ambient conditions. The phenotypes were classified as grades 0 (cold sensitive), 1, 2, 3, and 4 (cold tolerance). 


\section{Results}

In this study, parental Heigu (LTH) and Ha9366 plants were tested for germination at $15{ }^{\circ} \mathrm{C}$. Heigu started to germinate on the fourth day; the germination rate reached $30.4 \%, 77.7 \%$, and $88.9 \%$ on the fifth, sixth, and seventh day, respectively. Ha068143 started germinating on the fifth day at $15{ }^{\circ} \mathrm{C}$, and the germination rates reached $4.5 \%$ and $30.2 \%$ on the sixth and seventh day, respectively. The low-temperature germination rate of the parents reached a very significant level (Fig. 1). The low-temperature germination of the recombinant inbred line population at $15{ }^{\circ} \mathrm{C}$ had a distribution range of $0-90.8 \%$ on the fifth day, with an average value of $24.1 \%$, and a distribution range between 2.0 and $95.9 \%$ on the sixth day, with an average value of $49.9 \%$, while the distribution range was $13.7-99.0 \%$, with an average value of $69.2 \%$. Overall, it showed an approximately normal distribution and a strong separation of super-parents (Fig. 2).

QTL distribution and effects of cold-tolerance germination in populations

A total of four QTLs for cold tolerance of rice at the germination stage were detected by the MapQTL 6.0 software on the fifth, sixth, and seventh day which were distributed on chromosomes 3 and 12 of rice. The QTLs of $q L T G-12.5 d, q L T G-12.6 d$, and $q L T G-12.7 d$ were detected between the marker range of RM415 and RM453 on chromosome 12, which improved cold tolerance at the germination stage on the fifth, sixth, and seventh days. The interval size was $2.48 \mathrm{cM}$, and

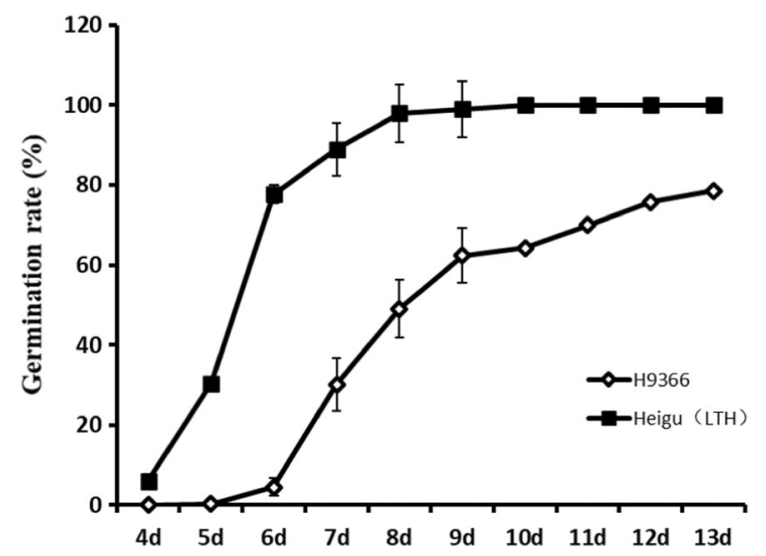

Fig. 1 Differences in germination rates between parental lines at $15{ }^{\circ} \mathrm{C}$
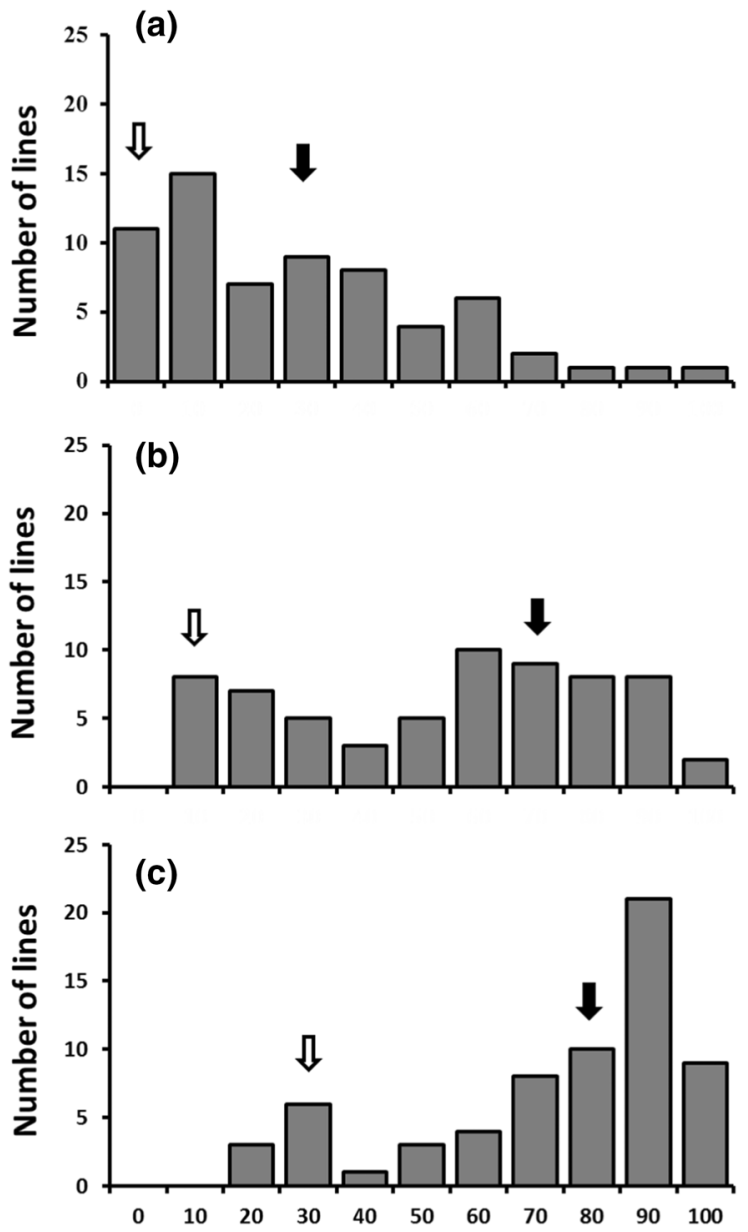

Fig. 2 Frequency distributions for low-temperature germination in RILs. A Experimental treatment for 5 days. B Experimental treatment for 6 days. C Experimental treatment for 7 days. Black and white arrows indicate the mean values of LTH and $\mathrm{H} 9366$, respectively

the LOD values were $4.22,4.05$, and 4.12 , respectively. The PVE values reached $25.9 \%, 24.9 \%$, and $21.3 \%$, respectively. This QTL enhanced the lowtemperature tolerance of rice during germination when it was present in the Ha 9366 allele. On the eighth day of low-temperature treatment, we detected a QTL on chromosome 3, with LOD of 4.09, PVE of $22.1 \%$, and improved the cold tolerance of rice at the germination stage when present in the Heigu allele (Table 1; Figs. 4 and 5).

The solid line represents the LOD value of germination rate on the fifth day at $15{ }^{\circ} \mathrm{C}$; the double line represents the LOD value of germination rate on the sixth day at $15{ }^{\circ} \mathrm{C}$; the dashed line represents the LOD 


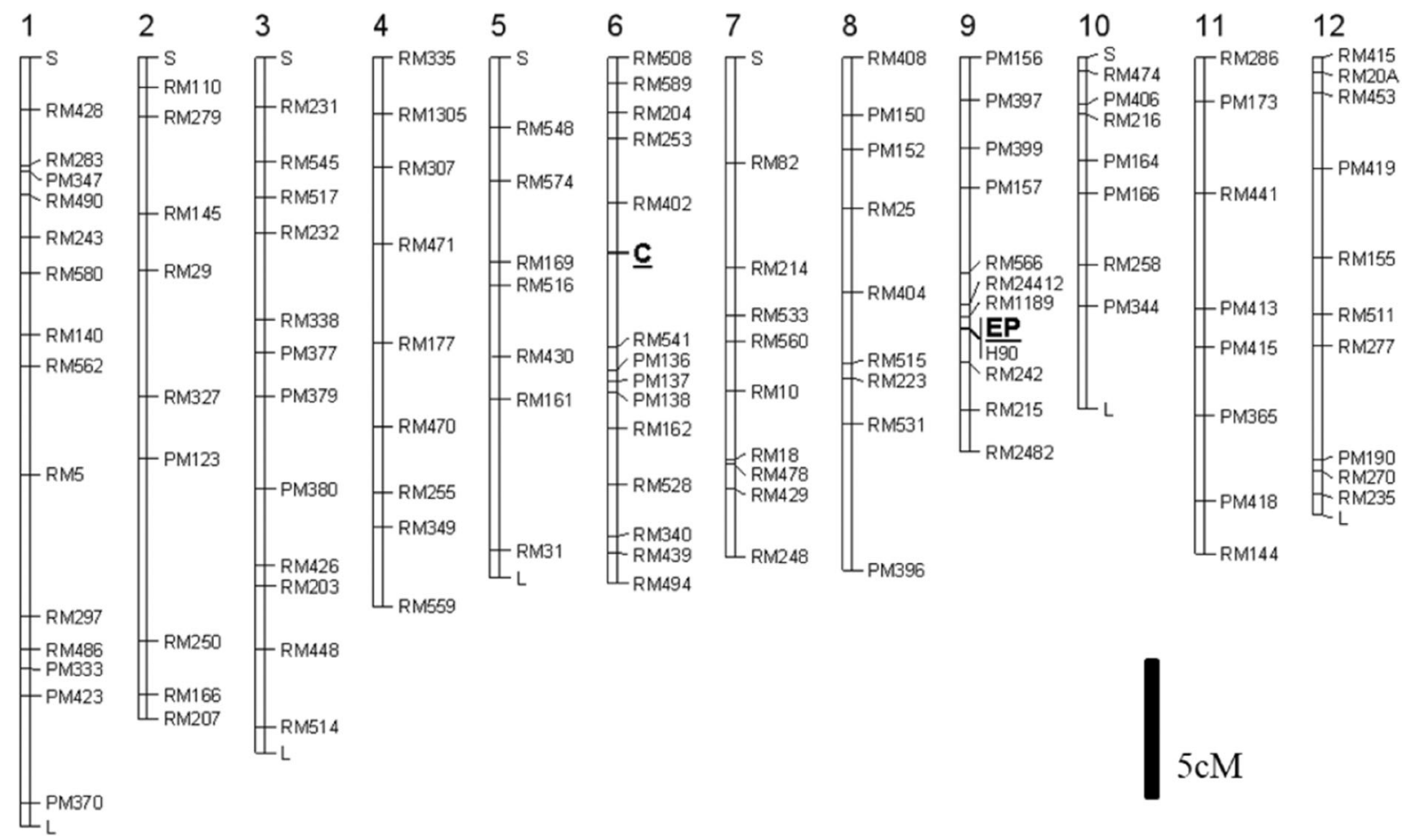

Fig. 3 The linkage mapping of recombinant inbred line populations between Heigu and Ha 9366

value of germination rate on the seventh day at $15^{\circ} \mathrm{C}$; the dotted line represents the standard LOD value calculated by permeation test $(P \leq 0.005)$.

The solid line represents the LOD value of germination rate on the fifth day at $15^{\circ} \mathrm{C}$; the double line represents the LOD value of germination rate on the sixth day at $15{ }^{\circ} \mathrm{C}$; the dashed line represents the LOD value of germination rate on the seventh day at $15^{\circ} \mathrm{C}$; the dotted line represents the standard LOD value calculated by permeation test $(P \leq 0.005)$.

Validation of cold tolerance QTL and analysis of interaction effects during germination

We validated the detected cold tolerance QTLs during the germination period and evaluated the interaction effects of these QTLs. First, the genotypes of qLTG-3 and qLTG-12, which govern cold tolerance at the germination stage, were distinguished, and a combination of two QTL sites and four alleles were selected from the recombinant inbred lines ( $q L T G-3$ is the allele of H9366, $q L T G-12$ is also the allele of LTH; b: $q L T G-3$ is the allele of LTH, $q L T G-12$ is the allele of LTH; c: $q L T G-3$ is the allele of H9366, $q L T G-12$ is also the allele of H9366; $\mathrm{d}$ : $q L T G-3$ is the allele of LTH allele, and $q L T G-12$ is also the allele of H9366). Among the 94 recombinant inbred lines, there were 36 strains in category a, 17 strains in category b, 29 strains in category c, and 12 strains in category d. The $q L T G$ 12 locus exhibited excellent low-temperature tolerance on the fifth, sixth, and seventh days of germination. The germination rates at different time points (five, six, and seven days) are shown in Fig. 6. The germination rate of category $c$ was $16-27 \%$ higher than that of category a, and that of category $d$ was 20-36\% higher than category b, both reached a significant difference. Moreover, the results of the two-way ANOVA showed that the F values of $q L T G$ - 
Chr. 3

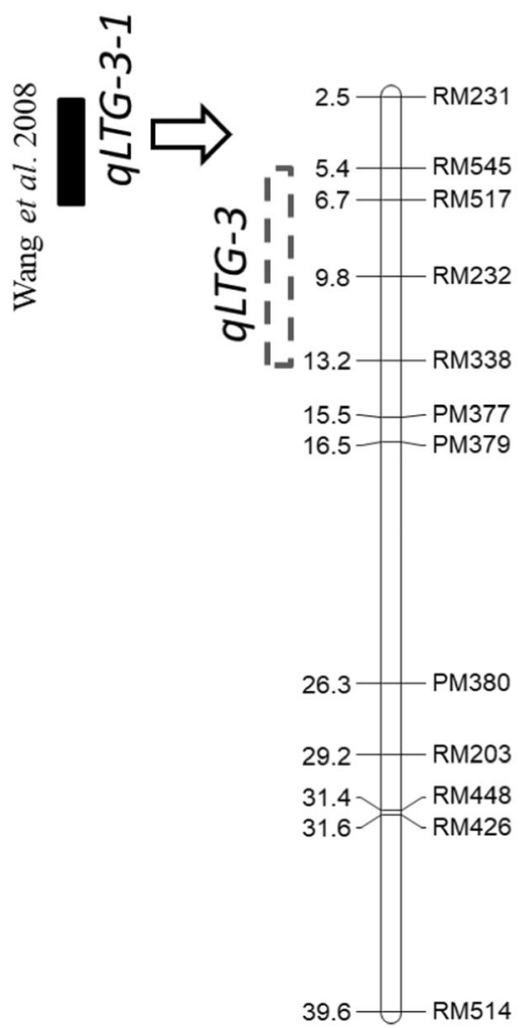

LOD

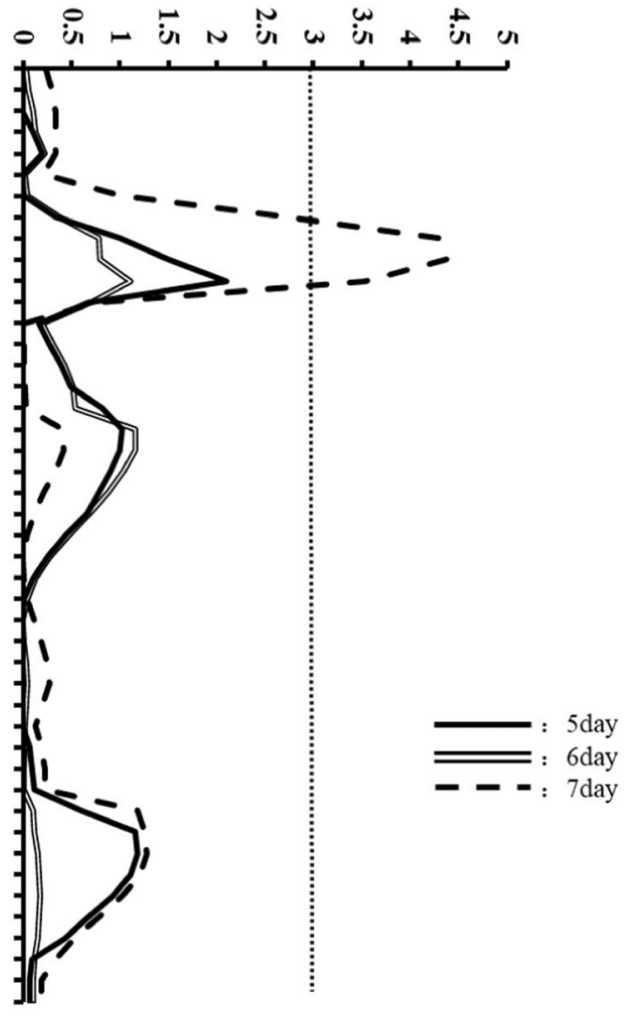

Fig. 4 The germination stage tolerance gene QTL-qLTG-3 is located on chromosome 3

12 reached 10.78 to 14.81 on the fifth, sixth, and seventh days and the significance reached over $0.1 \%$ (Fig. 6; Table 2). The qLTG-3 locus showed no significant effect on the germination rate on day 5 and day 6 , and only showed a significant difference for the germination rate on day 7 ; the $F$ value and significance were 4.52 and $5 \%$, respectively. The HTL allelic type effectively increased the germination rate from 4 to $8 \%$ under low-temperature conditions in this population (Fig. 6; Table 2). The above results suggested that the interaction effects between the QTLs, which were detected for the cold-tolerant recombinant inbred lines during germination, were mainly additive.

Verification of cold tolerance of qLTG-3 and qLTG-12 at the germination stage

Thirty-two rice lines suitable for cultivation in the Heilongjiang Province, China, were genotyped using the qLTG-3 and qLTG-12-related quantitative SSR primers RM232 and RM20A. Four allelic combinations were obtained (a: qLTG-3 was the H9366 allele 


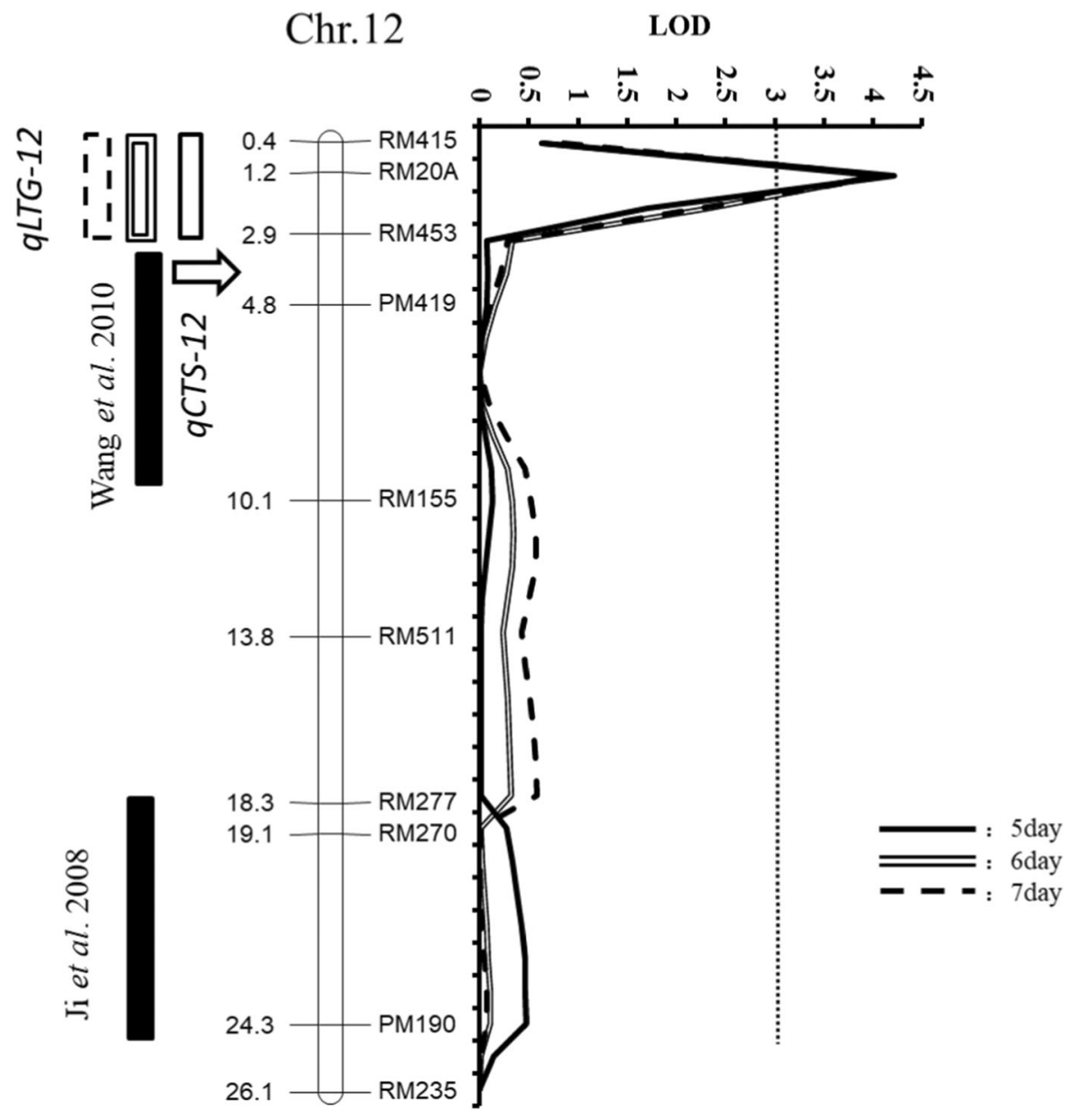

Fig. 5 Germination stage tolerance gene QTL-qLTG-12 is located on chromosome 12

and qLTG-12 was also the LTH allele; b: qLTG-3 was the LTH allele and qLTG-12 was the LTH allele; c: qLTG-3 was the H9366 allele and qLTG-12 was the H9366 allele; d: qLTG-3 was the LTH allele and qLTG-12 was the H9366 allele) (Fig. 7). The cold resistance test at the germination stage was also conducted for the 32 lines. The 32 lines were distributed from grades 0 to 4 for cold resistance. According to the genotype classification, grade $\mathrm{d}$ had the best cold tolerance, with a mean value of 3.7 , which was significantly higher than grades a, b, and c. The mean values for cold tolerance in grades $a$ and $b$ were 0.7 and 1.4, respectively, and the difference between the two grades (0.7) was not significant (Fig. 8).

\section{Discussion}

It is well-known that direct-sown rice can reduce the time and cost of growing rice, but it is difficult to apply this method in the northernmost areas of rice cultivation, such as northern China and Hokkaido, Japan, where the temperature is a limiting factor. Many QTLs related to low temperature at the germination stage have been reported. However, there is a lack of information on the actual molecular marker-assisted breeding among the QTLs.

Two QTLs associated with low-temperature germination, i.e., qLTG-3 and qLTG-12, were detected in this study. The qLTG-3 locus was located near the qLTG-3.1 locus, as reported by Funino (2008). Thus, 


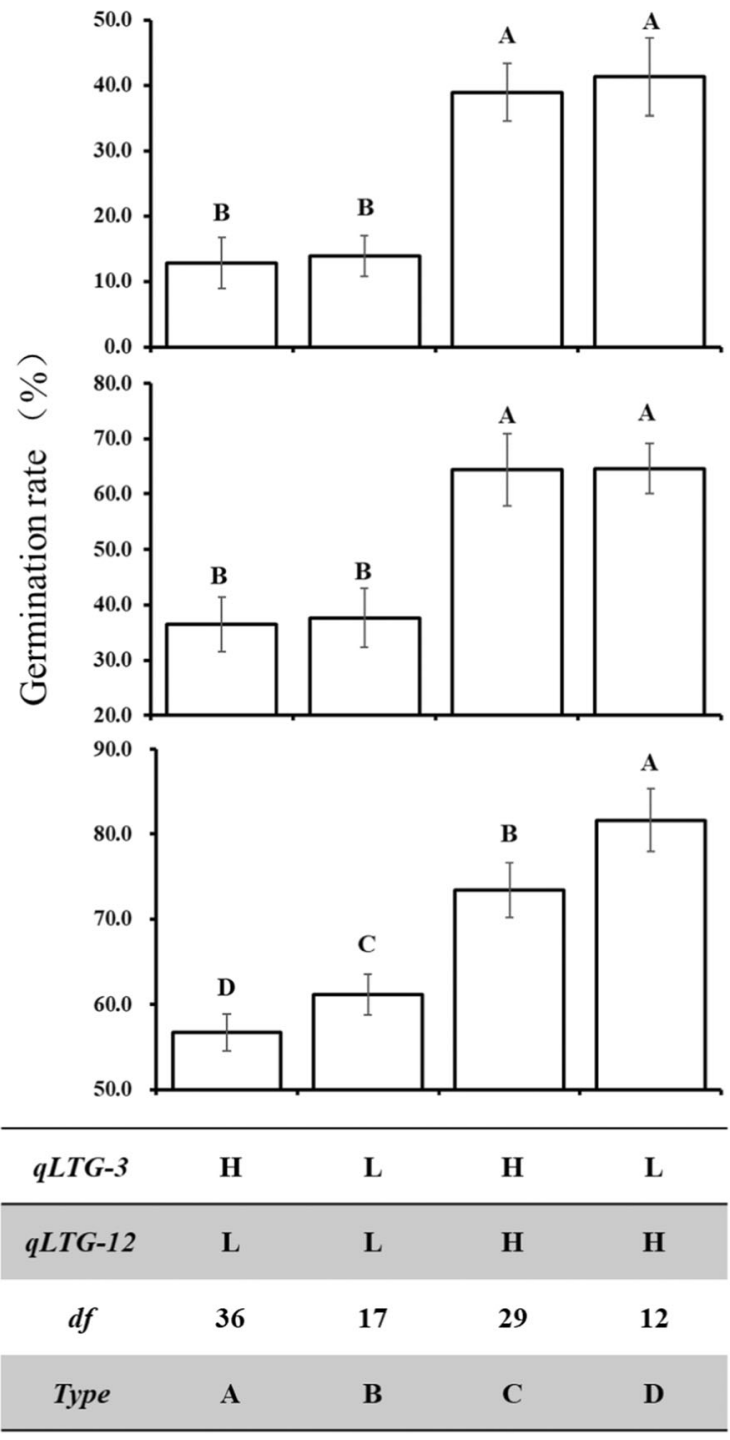

Fig. 6 Interactions between two QTLs related to low-temperature germination on chromosomes 3 (qLTG-3) and 12 (qLTG12). (I) The 5-day germination rate at $15{ }^{\circ} \mathrm{C}$, (II) The 6-day germination rate at $15^{\circ} \mathrm{C}$, (III) The 7-day germination rate at $15{ }^{\circ} \mathrm{C}$. The alleles of the QTLs were estimated by their flanking markers (Table 2). L and $\mathrm{H}$ represent alleles of LTH and Ha9366, respectively. The bar plots show germination rate (\%) \pm SD. Different letters above the bars indicate a significant difference based on the Tukey-Kramer test

qLTG-3, detected on chromosome 3 in this study, was probably influenced by the same gene as qLTG-3.1. The qLTG-3.1 locus is the main QTL controlling germination at low temperatures and can substantially increase the germination rate under low-temperature conditions. The main QTL locus detected in this study was qLTG-12. Chromosome 12 is the least reported of the many QTLs for low-temperature germination, with only three studies providing evidence of its role ( $\mathrm{Ji}$ et al. 2007; Hou et al. 2004; Yao et al. 2020). The qLTG-12 locus detected in this study was different from the three previously reported loci and can be considered to be a novel QTL for the study on cold tolerance at the germination stage in rice. Reciprocal effects are important and can improve molecular breeding in crops, while research on the supernumerary reciprocity in low-temperature germination of rice is lacking (Zhang et al. 2005; Ji et al. 2008). In this study, we detected a QTL (qLTG-12) that possessed a strong epistatic interaction effect with the main lowtemperature tolerance germination QTL (qLTG-3.1). A QTL locus with cold tolerance was reported near qLTG-12. The gene controlling qCTS-12 was OsGSTZ2, and Kim et al. (2011) found that the SNP locus in the OsGSTZ2 gene resulted in amino acid differences that were significantly associated with low-temperature sensitivity in rice seedlings. Additionally, Takesawa et al. (2020) reported that OsGSTZ2, when overexpressed, improved germination under low-temperature conditions and low-temperature tolerance at the seedling stage. In this study, qLTG-3 and qLTG-12 effectively improved cold tolerance at the seedling stage. Therefore, it is likely that the qLTG-12 locus detected in this study was due to the effect of OsGSTZ2.

In this study, a QTL (qLTG-12) was detected on chromosome 12 with a reciprocal effect with the main QTL (qTLG-3.1) for cold tolerance at germination. The double locus improved cold tolerance at the germination stage by 22-27\%, overall. Also, qLTG-12 improved the cold tolerance of rice at the germination stage and the seedling stage. The results of this study were therefore well suited to molecular markerassisted breeding and provided the materials and the molecular markers for the germination traits at low temperatures. 
Table 2 Results of MOM mapping for Low-Temperature Germination (LTG) in the RILs

\begin{tabular}{lclllll}
\hline Experimental treatment days & Chromosome & Interval marker & QTL & LOD & PVE (\%) & Additive effect \\
\hline 5 & 12 & $R M 415-R M 453$ & $q L T G-12$ & 4.22 & 25.90 & -11.99 \\
6 & 12 & $R M 415-R M 453$ & $q L T G-12$ & 4.05 & 24.90 & -14.16 \\
7 & 3 & $R M 517-R M 338$ & $q L T G-3$ & 4.09 & 22.10 & 14.043 \\
& 12 & $R M 415-R M 453$ & $q L T G-12$ & 4.12 & 21.30 & -12.15 \\
\hline
\end{tabular}

LOD values, PVE (phenotypic variance explained by each QTL), and additives effect are listed. Negative values of additive effect indicated the effect increasing trait values by the LTH alleles, respectively

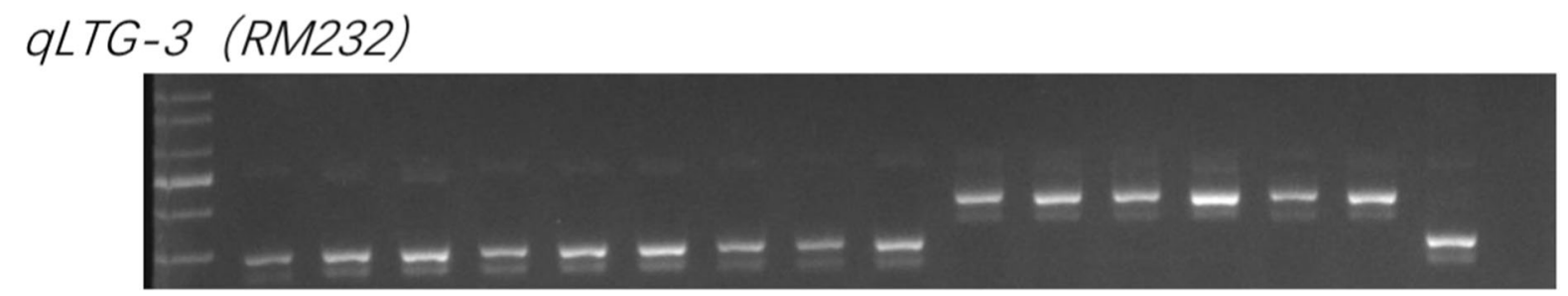

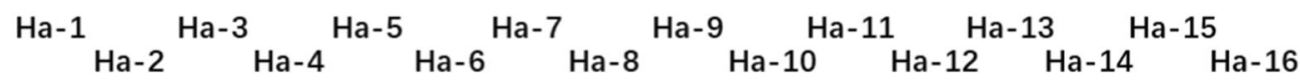

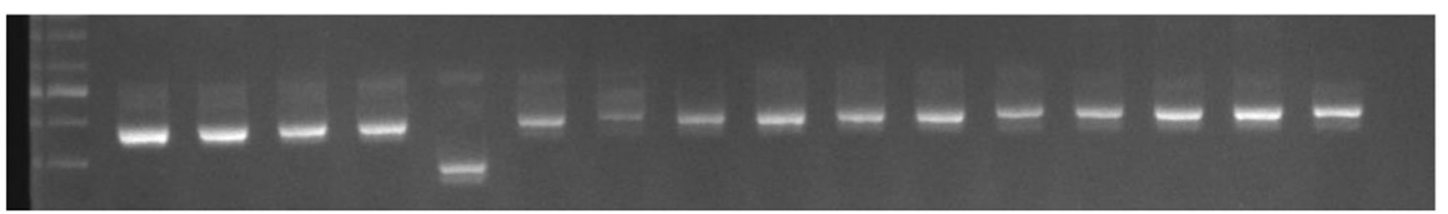

Нa-17 Нa-19 Нa-21 Нa-23 Нa-25 Нa-27 Нa-29 На-31 На-18 На-20 На-22 На-24 На-26 На-28 На-30 На-32

$q L T G-12$ (RM20A)
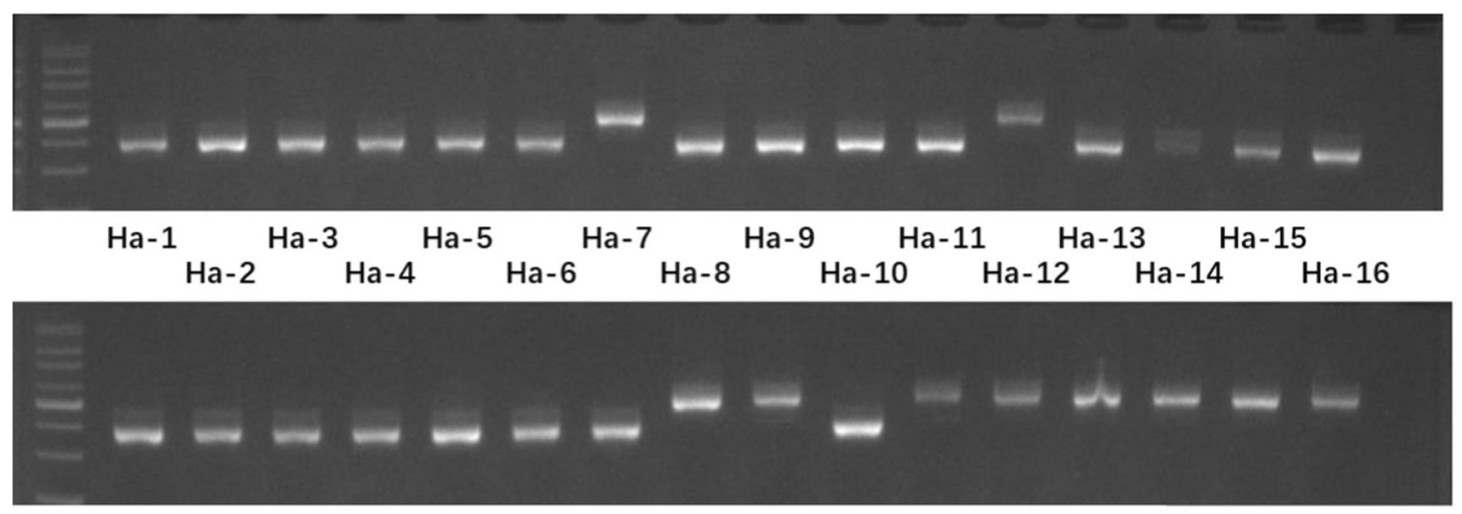

Ha-17 Нa-19 Нa-21 Ha-23 Ha-25 Нa-27 Нa-29 На-31 Ha-18 Ha-20 Ha-22 Ha-24 Ha-26 Ha-28 Ha-30 Нa-32

Fig. 7 Genotypic analysis of 32 rice lines using qLTG-3 and qLTG-12 


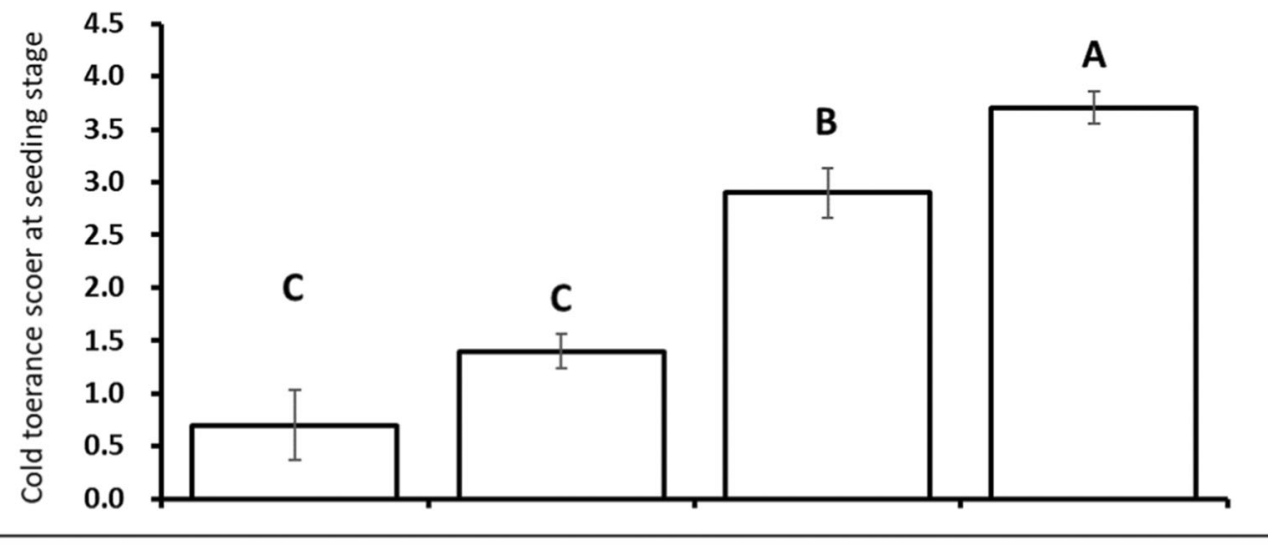
$q L T G-3$
H
L
H
L

$q L T G-12$

No. of lines

Type
$\mathbf{L}$

3

a
L

8

b
H

9

c
H

12
Fig. 8 Interactions between two QTLs for the low-temperature seeding stage on chromosomes 3 ( $q L T G-3)$ and 12 ( $q L T G-12)$. L and $\mathrm{H}$ indicate the LTH and Ha9366 alleles, respectively. The bar plots show the germination rate $(\%) \pm \mathrm{SD}$. Different letters above the bars indicate a significant difference based on the Tukey-Kramer test

Table 3 Two-way ANOVA analysis for the effects of one QTL and one gene for Low-Temperature Germination (LTG) in the RILs

\begin{tabular}{|c|c|c|c|c|c|c|c|c|c|c|}
\hline \multirow[t]{2}{*}{ Factor } & \multirow[t]{2}{*}{ d.f } & \multicolumn{3}{|l|}{5 Day } & \multicolumn{3}{|l|}{6 Day } & \multicolumn{3}{|l|}{7 Day } \\
\hline & & $M S$ & $F$-value & $P$ & $M S$ & F-value & $P$ & $M S$ & F-value & $P$ \\
\hline$q L T G-3-1$ & 1 & 469.62 & 1.18 & $0.28 \mathrm{~ns}$ & 820.20 & 1.46 & $0.233 \mathrm{~ns}$ & 1964.44 & 4.52 & $0.046 *$ \\
\hline$q L T G-12$ & 1 & 5895.32 & 14.81 & $0.00 * * *$ & 6419.30 & 11.42 & $0.001 * * *$ & 4789.21 & 10.78 & $0.002 * *$ \\
\hline$q L T G-3-1 \times q L T G-12$ & 1 & 1406.45 & 3.53 & $0.07 \mathrm{~ns}$ & 2917.43 & 5.19 & $0.027 *$ & 2045.12 & 4.60 & $0.037 *$ \\
\hline Error & 94 & 398.07 & & & 562.26 & & & 444.16 & & \\
\hline
\end{tabular}

$*, * *$ and $* * *$ indicated the statistical significances at $5 \%, 1 \%$ and $0.1 \%$ levels, respectively

\section{Conclusions}

We used recombinant inbred lines LTH and H9366 to locate the quantitative trait loci related to lowtemperature germination of rice. The two QTL sites (qLTG-3 and qLTG-12) were located on chromosomes 3 and 12. QTL accounted for 21.3-25.9\% of the phenotypic variations at three germination time points within the two QTLs. The reciprocal effects between the two QTL sites were also detected. In this population, double QTLs increased the germination rate by $22-27 \%$. Moreover, qLTG-12 enhanced cold tolerance at the germination stage and the seedling stage. This study provided the materials and molecular markers for molecular marker-assisted breeding of cold tolerance. 
Author contributions In this paper, LC is mainly responsible for the overall experimental progress and data collation, QTL analysis and paper writing. SS is mainly responsible for the overall thinking of the paper and the revision of the paper. GD, TW were mainly responsible for the investigation of phenotypic data. LB, JZ were in charge of rice field management. YL is responsible for laboratory management, TX, HJ and KL are responsible for scientific funding management, and XW and GY are responsible for molecular experiments.

Funding This work was supported by the Provincial Science Foundation Project of Heilongjiang (LC2018013); Heilongjiang academy of agricultural sciences outstanding youth project (2021JCQN001); Supported by the National Key Research and Development Program of China (2017YFD0300406)Heilongjiang Provincial Scientific Institution Basal Research Fund (CZKYF2020A001); Provincial Postdoc Program of Heilongjiang (LBH-Z17199); National Key Research and Development Scheme Project (2017YFD0300501); National Key Research and Development Scheme Project (2017YFD0100503); Key Program of Heilongjiang Applied Technology Research and Development Scheme (GA18B101); Special Foundation for National Modern Agricultural Industry Technology System (CARS-01-57); Academic Project of Heilongjiang Academy of Agricultural Sciences (2019JJPY008); Academic Project of Heilongjiang Academy of Agricultural Sciences (2020FJZX018); Supported by the earmarked fund for China Agriculture Research System. Heilongjiang Academy of Agricultural Sciences 2020 Institution-level introduction and Innovation Project (2020YJ001).

Data availability All data generated or analyzed during this study are included in this published article.

Code Availability Not applicable.

\section{Declarations}

Conflict of interest The authors declare that they have no conflict of interest.

Open Access This article is licensed under a Creative Commons Attribution 4.0 International License, which permits use, sharing, adaptation, distribution and reproduction in any medium or format, as long as you give appropriate credit to the original author(s) and the source, provide a link to the Creative Commons licence, and indicate if changes were made. The images or other third party material in this article are included in the article's Creative Commons licence, unless indicated otherwise in a credit line to the material. If material is not included in the article's Creative Commons licence and your intended use is not permitted by statutory regulation or exceeds the permitted use, you will need to obtain permission directly from the copyright holder. To view a copy of this licence, visit http://creativecommons.org/licenses/by/4.0/.

\section{References}

Cao W et al (2018) Evaluation of seed germination vigor of rice cultivars under low temperature or hypoxic condition related with direct seeding rice. Mol Plant Breed 16:3259-3268

Chen W, Li W (2005) Mapping of QTL conferring cold tolerance at early seedling stage of rice by molecular markers. J Wuhan Bot Res 23:116-120

Chen L, Lou QJ, Sun ZX, Xing YZ, Luo YJ (2006) QTL mapping of low temperature germinability in rice. Chin J Rice Sci 20:159-164

Farooq M, Siddique KH, Rehman H, Aziz T, Lee D-J, Wahid A (2011) Rice direct seeding: experiences, challenges and opportunities. Soil Tillage Res 111:87-98

Feng YJ, Wang Q, Zhao HL, Song QL, Sun Y, Zeng XN (2020) Research status and prospect of the direct seeding technology of rice in China. China Rice 26:23-27

Fujino K et al (2004) Mapping of quantitative trait loci controlling low-temperature germinability in rice (Oryza sativa L.). Theor Appl Genet 108:794-799

Fujino K, Sekiguchi H, Matsuda Y, Sugimoto K, Ono K, Yano M (2008) Molecular identification of a major quantitative trait locus, qLTG3-1, controlling low-temperature germinability in rice. Proc Natl Acad Sci 105:12623-12628

Gong YJ, Xun X, Dong Y, Lin D, Ye S, Zhang X (2009) QTL Analysis of cold tolerance for two bud traits in rice. Mol Plant Breed 7:273-278

Han L et al (2007) Identification of quantitative trait loci for cold response of seedling vigor traits in rice. J Genet Genom 34:239-246

Hou M, Wang C, Jiang L, Wan J, An J (2004) QTL mapping and genetic analysis of rice germination ability at low temperature. Acta Genet 701-706

Ji S, Jiang L, Wang Y, Liu S, Liu X, Zhai H, Wan J (2007) Detection of genetic motifs for quantitative traits of lowtemperature germination tolerance in rice using backcross recombinant self-incompatible populations. J Nanjing Agric Univ 1-6

Ji S-L et al (2008) QTL and epistasis for low temperature germinability in rice. Acta Agron Sin 34:551-556

Jiang L, Liu S, Hou M, Tang J, Chen L, Zhai H, Wan J (2006) Analysis of QTLs for seed low temperature germinability and anoxia germinability in rice (Oryza sativa L.). Field Crops Res 98:68-75

Jiang N et al (2017) Mapping QTL for seed germinability under low temperature using a new high-density genetic map of rice. Front Plant Sci 8:1223

Kim SI, Andaya VC, Tai TH (2011) Cold sensitivity in rice (Oryza sativa L.) is strongly correlated with a naturally occurring I99V mutation in the multifunctional glutathione transferase isoenzyme GSTZ2. Biochem J 435:373-380

Li H-W, Zang B-S, Deng X-W, Wang X-P (2011) Overexpression of the trehalose-6-phosphate synthase gene OsTPS1 enhances abiotic stress tolerance in rice. Planta 234:1007-1018

Lin J, Zhang Y-d, Zhu Z, Zhao L, Chen T, Wang C-1 (2008) Detection and analysis of QTLs for cold tolerance at early seedling stage in rice (Oryza sativa $\mathrm{L}$.) using recombinant inbred lines. Acta Agric Jiangxi 20:1-3 
Murray MG, Thompson WF (1980) Rapid isolation of high molecular weight plant DNA. Nucleic Acids Res $8: 4321-4325$

Najeeb S et al (2020) Identification of main-effect quantitative trait loci (QTLs) for low-temperature stress tolerance germination-and early seedling vigor-related traits in rice (Oryza sativa L.). Mol Breed 40:10

Olajumoke B, Juraimi AS, Uddin M, Husni MH, Alam M (2016) Competitive ability of cultivated rice against weedy rice biotypes: a review. Chile J Agric Res 76:243-252

Qiao Y-L, Han L-Z, An Y-P, Zhang Y-Y, Cao G-L, Koh H-J (2005) Molecular mapping of QTLs for cold tolerance at the bud bursting period in rice. Sci Agric Sin 38:217-221

Schläppi MR et al (2017) Assessment of five chilling tolerance traits and GWAS mapping in rice using the USDA minicore collection. Front Plant Sci 8:957

Singh S, Sharma S, Prasad R (2001) The effect of seeding and tillage methods on productivity of rice-wheat cropping system. Soil Tillage Res 61:125-131

Su-Lan J et al (2008) QTL and epistasis for low temperature germinability in rice. Acta Agron Sin 34:551-556

Takesawa T (2002) Over-expression of $\zeta$ glutathione S-transferase in transgenic rice enhances germination and growth at low temperature. Mol Breed 9:93-101

Teng S, Zeng D, Qian Q, Huang D, Zhu H (2001) Location analysis of QTL for germinability in rice under low temperature. Chin Sci Bull 46:1104-1108

Ya ZJ, Lu SC, Hou K (2020) Development status, problems and application prospects of dry direct seeding rice. Crops 2:9-15
Yang T et al (2016) Identification and pyramiding of QTLs for cold tolerance at the bud bursting and the seedling stages by use of single segment substitution lines in rice (Oryza sativa $\mathrm{L}$.). Mol Breed 36:1-10

Yang L et al (2018) Identification of QTLs controlling lowtemperature germinability and cold tolerance at the seedling stage in rice (Oryza Sativa L.). Euphytica 214:1-17

Yao X-Y, Xiong Y-H, Huang Y-P, Wu Y-S, Yang P, Liu J, Yin J-H (2020) QTL Mapping for rice seed vigor under low temperature environment. Acta Agric Univ Jiangxiensis (Nat Sci Ed) 42:633-640

Zhang Z-H, Yu S-B, Yu T, Huang Z, Zhu Y-G (2005) Mapping quantitative trait loci (QTLs) for seedling-vigor using recombinant inbred lines of rice (Oryza sativa L.). Field Crops Res 91:161-170

Zhang XJ, Lai YC, Wang JH, Meng Y, Tang A, Dong WJ, Leng CX (2015) Development situation and countermeasures of rice direct seeding cultivation in heilongjiang province. Hlongjiang Agricultural Ences

Zhen MM (2015) The effect of seed priming on germination, growth performance and yield of dry direct-seeded rice. Huazhong Agriculture University

Publisher's Note Springer Nature remains neutral with regard to jurisdictional claims in published maps and institutional affiliations. 